Article

\title{
Simple Harmonic Oscillator Canonical Ensemble Model for Tunneling Radiation of Black Hole
}

\author{
Jinbo Yang, Tangmei He and Jingyi Zhang * \\ Center for Astrophysics, Guangzhou University, GuangZhou 510006, China; \\ 2111419011@e.gzhu.edu.cn (J.Y.); hetangm@gzhu.edu.cn (T.H.) \\ * Correspondence: zhangjy@gzhu.edu.cn \\ Academic Editor: Remo Garattini \\ Received: 30 August 2016; Accepted: 10 November 2016; Published: 23 November 2016
}

\begin{abstract}
A simple harmonic oscillator canonical ensemble model for Schwarzchild black hole quantum tunneling radiation is proposed in this paper. Firstly, the equivalence between canonical ensemble model and Parikh-Wilczek's tunneling method is introduced. Then, radiated massless particles are considered as a collection of simple harmonic oscillators. Based on this model, we treat the black hole as a heat bath to derive the energy flux of the radiation. Finally, we apply the result to estimate the lifespan of a black hole.
\end{abstract}

Keywords: PW tunneling; canonical ensemble; energy flux of Hawking radiation

\section{Introduction}

Since Hawking discovered that a black hole has thermal radiation in the 1970s, there has been an ongoing argument about whether Hawking radiation violates unitary principle in black hole physics, which is the so-called black hole information paradox [1-5].

In 2000s, Parikh and Wilzeck treated the emission of Hawking radiation as tunnelling-namely, out-going Hawking particles feel a barrier due to their self-gravity. Considering the effect of backreaction, which preserves the principle of energy conservation during tunneling, the spectra are not thermal spectra anymore. According to Parikh and Wilzeck's original result, the emission probability for a Schwarzchild black hole is

$$
\Gamma \propto e^{-8 \pi M \omega+4 \pi \omega^{2}}=\exp (\Delta S),
$$

in which $\Delta S$ is the increment of entropy. They claimed that the unitary principle is preserved according to this result [6-8].

The Parikh-Wilczeks (PW) tunneling framework has been applied for other black holes. From then on, the tunneling rate is generally proportional to $\exp (\Delta S)$ for different black holes, such as RN black hole, Kerr black hole, and Kerr-Newman black hole, etc. Meanwhile, the emission of charged massive particles is also investigated [9-31].

It is interesting that the following formula always appears in the calculation

$$
d S=\frac{1}{T}\left(d M-V_{+} d Q-\Omega_{+} d J\right),
$$

which implies that the tunneling is equivalent to a quasi-static process. Of course, a quasi-static process is reversible, while an arbitrary thermodynamical process is not. Once a non-quasi-static process is considered, the information should be lost. This argument was proposed in Reference [18], and it inspires us to apply a canonical ensemble method for tunneling model. 
In a traditional fashion, a cannonical ensemble is derived in the following way. Considering that the system interacts with a huge heat bath, they exchange energy with each other and constitute an isolated system. According to the principle of equivalent possibility, the probability of the system in $s$ state is

$$
P_{\mathcal{S}} \propto \Omega_{B H}\left(E_{t o t}-E_{S}\right) .
$$

Since $E_{s} \ll E_{t o t}$, take Taylor expansion,

$$
\begin{aligned}
\ln \Omega_{B H}\left(E_{t o t}-E_{S}\right)= & \ln \Omega_{B H}\left(E_{t o t}\right)+\left(\frac{\partial \ln \Omega_{B} H}{\partial E}\right)_{E=E_{t o t}}\left(-E_{S}\right)+ \\
& \frac{1}{2}\left(\frac{\partial^{2} \ln \Omega_{B H}}{\partial E^{2}}\right)_{E=E_{t o t}}\left(-E_{S}\right)^{2}+\cdots
\end{aligned}
$$

Keep the leading term, and define

$$
\beta=\left(\frac{\partial \ln \Omega}{\partial E}\right)_{E=E_{t o t}}
$$

Then, Expression (3) becomes

$$
P_{S} \propto \exp \left(-\beta E_{S}\right) .
$$

This is a so-called canonical ensemble. It is worth noting that we only take the first order in Taylor expansion here. It becomes interesting when the second order is also considered. Let us define the following parameter

$$
\lambda=\frac{1}{2}\left(\frac{\partial^{2} \ln \Omega}{\partial E^{2}}\right)_{E=E_{t o t}}=-\frac{k_{B} \beta^{2}}{2 C_{B H}},
$$

in which $C_{B H}$ is the heat capacity of a black hole and $k_{B}$ is the Boltzmann constant. Then, the expansion up to second order is

$$
\ln \Omega_{B H}=-\beta E_{S}+\lambda E_{S}^{2} .
$$

For a Schwarzchild black hole, $\beta=8 \pi M$ and $\lambda=4 \pi$ (in Planck units), and $\lambda$ is a constant. Thus,

$$
P_{S} \propto e^{-8 \pi M E_{S}+4 \pi E_{s}^{2}}
$$

The above formula for the probability of $s$ state is similar to the tunneling rate given by Parikh-Wilzeck's framework. It sheds light on the calculation of the corrected average energy flux and the corrected lifespan of black hole. Generally, the lifespan of a black hole is calculated through the Stefan-Boltzmann law because of the pure black-body feature of Hawking radiation. However, in Parikh-Wilczek's tunneling model, Hawking radiation spectra are not pure thermal spectra anymore. So, the average energy flux should also be corrected. Then, the flux would lead to the correction for the lifespan of black holes.

There may be a question about which ensemble should be chosen for other black holes. Let us take the emission of charged massive particles from a Kerr black hole as an example. A generalized $\mathrm{PW}$ model is studied in [20] and [27]. The result is still $\Gamma \propto \exp (\Delta S)$, but $\Delta S$ looks more complex,

$$
\Delta S=2 \pi\left[(M-\omega)^{2}+(M-\omega) \sqrt{(M-\omega)^{2}-a^{2}}\right]-2 \pi\left[M^{2}+M \sqrt{M^{2}-a^{2}}\right] .
$$

In this situation, the whole energy and charge are conserved, but emissions take away part of them. Therefore, the energy and charge of the black hole are decreasing. It is natural to think about a grand canonical ensemble rather than a canonical ensemble. However, the concrete study of energy flux in this case is very difficult due to the complex expression of $\Delta S$.

This paper is organized as follows: in Section 2, we will talk about the simple harmonic oscillator (SHO) canonical ensemble model in detail. In Section 3, we will apply the result of Section 2 to estimate the lifespan of a black hole with initial mass $M$ and discuss. SI units rather than Planck units is used in the following content. 


\section{SHO Canonical Ensemble Model}

According to quantum field theory for free field, photons can be identified as excited quanta of an electromagnetic field's harmonic mode. The frequency of $\vec{p}$ mode is $\omega_{\vec{p}}=|p| / \hbar$. Treat the thermal system as a collection of harmonic modes. The energy of the radiation is

$$
E_{s}=\sum_{p} n_{p} \hbar \omega_{p}
$$

The partition function in first order is

$$
Z=\sum_{\left\{n_{\vec{p}}\right\}} e^{-\sum \vec{p} \beta n_{\vec{p}} \hbar \omega_{\vec{p}}}
$$

where $\left\{n_{\vec{p}}\right\}$ presents the distribution of particles. It means that we sum over all order set $\left\{n_{1}, n_{2}, n_{3}, \ldots, n_{i}, \ldots\right\}$ in which index $i$ runs over the whole momentum space. It is obvious that $Z$ can also be expressed as

$$
\sum_{\left\{n_{\vec{p}}\right\}} \prod_{\vec{p}} e^{-\beta n_{\vec{p}} \hbar \omega_{\vec{p}}}=\prod_{\vec{p}} \sum_{n_{\vec{p}}} e^{-\beta n_{\vec{p}} \hbar \omega_{\vec{p}}}
$$

Define $Z_{\vec{p}}=\sum_{n_{\vec{p}}} e^{-\beta n_{\vec{p}} \hbar \omega_{\vec{p}}}$, then $Z=\prod_{p} Z_{p}$. There is no mixture between different modes. Thus, for an arbitrary mode, the average particle number is

$$
\bar{n}=\frac{\sum_{n=0}^{\infty} n e^{-\beta n \hbar \omega}}{\sum_{n=0}^{\infty} e^{-\beta n \hbar \omega}}=\frac{1}{e^{\beta \hbar \omega}-1} .
$$

This is the boson distribution.

In Parikh-Wilzeck's tunneling model, they treated the outgoing particle as s-mode. The s-mode should be thought of as a superposition of plane wave at large $r$ region. On one hand, the emission rate is $\Gamma \propto e^{-8 \pi G M \omega / c^{3}+4 \pi G \hbar \omega^{2} / c^{5}}$. On the other hand, considering second order in canonical ensemble, the probability of a particular $s$ state is $P_{S} \propto e^{-8 \pi G M E_{s} / \hbar c^{3}+4 \pi G E_{s}^{2} / \hbar c^{5}}$. Obviously, for one particle state $E_{s}=\hbar \omega$, the result is exactly the same with Parikh-Wilzeck's tunneling model. However, since $E_{s}=\sum_{\left\{n_{p}\right\}} n_{p} \hbar \omega_{p}$, the term $4 \pi G E_{s}^{2} / \hbar c^{5}$ mixes different modes. In order to simplify the calculation, the approximation that every mode is independent is considered in our model. Thus, the total partition function is still $Z=\prod_{p} Z_{p}$. For single harmonic mode, there is

$$
\sum_{n} e^{-\beta n \hbar \omega+\lambda n^{2} \hbar^{2} \omega^{2}}
$$

Average particle number $\bar{n}=\frac{\sum_{n=0}^{\infty} n e^{-\beta n \hbar \omega}}{\sum_{n=0}^{\infty} e^{-\beta n \hbar \omega}}=\frac{1}{e^{\beta \hbar \omega}-1}$ should be replaced by

$$
\bar{n}=\frac{\sum_{n=0}^{\infty} n e^{-\beta n \hbar \omega+\lambda n^{2} \hbar^{2} \omega^{2}}}{\sum_{n=0}^{\infty} e^{-\beta n \hbar \omega+\lambda n^{2} \hbar^{2} \omega^{2}} .}
$$

It is very difficult to calculate the average number, and there is one more serious problem; the sum (15) is divergence. Meanwhile, whether or not the average number (16) converges is still a problem. Obviously, Expression (15) needs regularization. This is an important issue. However, we want to find the asymptotic expansion of Expression (16) rather than the precise summation in this paper. It can be done in the following way. Define function

$$
\Xi(s, x)=\frac{\sum_{n=0}^{\infty} n e^{-n x+n^{2} s x^{2}}}{\sum_{n=0}^{\infty} e^{-n x+n^{2} s x^{2}} .}
$$


Expand the function with respect to variable $s$

$$
\Xi(s, x)=\Xi(0, x)+\left(\frac{\partial \Xi}{\partial s}\right)_{s=0}+O\left(s^{2}\right)=\frac{1}{e^{x}-1}+\frac{e^{2 x}+3 e^{x}}{\left(e^{x}-1\right)^{3}} x^{2} s+O\left(s^{2}\right) .
$$

Supposing that the black hole is a heat bath with minus heat capacity in flat spacetime, the corrected average energy flux can be calculated as

$$
j_{u}=\frac{2}{(2 \pi \hbar)^{3}} \int_{0}^{+\infty} d p_{x} \int_{-\infty}^{+\infty} d p_{y} \int_{-\infty}^{+\infty} d p_{z} \bar{n} p_{x} c^{2}
$$

in which $\bar{n}$ is irrelevant with direction. However, it depends on the value of momentum $p$. Integral under spherical coordinates,

$$
\begin{aligned}
j_{u} & =\frac{2 \pi k_{B}^{4} T^{4}}{(2 \pi \hbar)^{3} c^{2}} \int_{0}^{\infty} \Xi\left(\lambda k_{B}^{2} T^{2}, x\right) x^{3} d x \\
& =\frac{2 \pi k_{B}^{4} T^{4}}{(2 \pi \hbar)^{3} c^{2}} \int_{0}^{\infty} \frac{x^{3}}{e^{x}-1} d x+\lambda \frac{2 \pi k_{B}^{6} T^{6}}{(2 \pi \hbar)^{3} c^{2}} \int_{0}^{\infty} \frac{e^{2 x}+3 e^{x}}{\left(e^{x}-1\right)^{3}} x^{5} d x+O\left(\lambda^{2}\right)
\end{aligned}
$$

Finally, the result is

$$
j_{u}=\sigma T^{4}+\lambda \sigma \frac{4\left(\pi^{4}-45 \zeta(5)\right)}{9 \zeta(4)} k_{B}^{2} T^{6}+O\left(T^{8}\right),
$$

in which $\sigma$ is the Stefan-Boltzman constant and $\zeta(n)$ is Riemann zeta function. By the same method, the corrected flux for a massless fermion can also be calculated as follows:

$$
j_{u}=\frac{2 \pi k_{B}^{4}}{(2 \pi \hbar)^{3} c^{2}} \frac{7 \pi^{4}}{120} T^{4}+\frac{2 \pi k_{B}^{4}}{(2 \pi \hbar)^{3} c^{2}} \frac{2555 \pi^{10}}{66} \frac{4 \pi G}{\hbar c^{5}} k_{B}^{2} T^{6}+O\left(T^{8}\right) .
$$

\section{Lifespan of a Black Hole}

Once the corrected flux is known, the corrected lifespan of a black hole can also be calculated. Under a simple consideration of energy conservation, the increment of the black hole energy should equal to minus energy flow from it, which means

$$
c^{2} \frac{d M}{d t}=-j_{u} A
$$

The flux ignoring the higher order of $s=\lambda k_{B}^{2} T^{2}$ is expressed as

$$
j_{u}=\sigma T^{4}+\frac{4 \pi G}{\hbar c^{5}} \sigma \frac{4\left(\pi^{4}-45 \zeta(5)\right)}{9 \zeta(4)} k_{B}^{2} T^{6} .
$$

Assuming that the surface area of radiation is coincident with horizon area, and substituting the temperature of Hawking radiation $T=\hbar c^{3} / 8 \pi k_{B} G M$ and values of all parameters into Equation (23), we obtain

$$
\begin{aligned}
\frac{d M}{d t} & =-\frac{16 \pi G \sigma}{c^{6} M^{2}}\left(\frac{\hbar c^{3}}{8 \pi G k_{B}}\right)^{4}\left(1+\frac{4\left(\pi^{4}-45 \zeta(5)\right)}{9 \zeta(4)}\left(\frac{\hbar c^{3}}{8 \pi G k_{B}}\right)^{2} \frac{4 \pi G k_{B}^{2}}{\hbar c^{5} M^{2}}\right) \\
& =-\frac{3.90 \times 10^{15}}{M^{2}}\left(1+\frac{2.04 \times 10^{-16}}{M^{2}}\right) .
\end{aligned}
$$

The lifespan is found as the following when the above equation is solved.

$$
t=\frac{10^{-23}}{1.23} \times\left(\frac{M^{3}}{3}-2.04 \times 10^{-16} M+\left(2.04 \times 10^{-16}\right)^{\frac{3}{2}} \arctan \left(\frac{M}{\sqrt{2.04 \times 10^{-16}}}\right)\right),
$$


in which $M$ is the initial mass of the black hole.

Our result shows that the lifespan correction is extremely small. Treating billion sun mass as initial mass for black hole, and taking it as a concrete example to estimate the lifespan, we find that the result from the uncorrected Stefan-Boltzmann's law is $3 \times 10^{93}$ years, but the correction is less than two years! However, it is still significant to have a systematic method by which the correction can be calculated order by order.

\section{Discussion}

The similarity between PW's result and (grand) canonical ensemble is remarkable. This led to an order by order estimation of energy flux and black hole lifespan. However, it is not new to find high order correction of Hawking radiation energy flux. Modak [32] has also constructed an order by order calculation for energy flux of Hawking radiation. They studied the energy flux by the following integral

$$
\langle E\rangle=\frac{1}{2 \pi} \int_{0}^{\infty}\left\langle n_{\omega}\right\rangle \omega d \omega
$$

In our point of view, the degenerate degree of every energy level is less considered in their method. On the contrary, the barrier for high angular momentum is less considered in our method. One can expect that the precise flux should be larger than their result, but smaller than ours.

On the other hand, Modak [32] studied backreaction at the quantum level. They thought that redefining $\kappa(M)=\frac{1}{4 M} \rightarrow \kappa(M-n \hbar \omega)=\frac{1}{4(M-n \hbar \omega)}$ would account for the effect of backreaction. The quantum state is

$$
|\psi\rangle=N \sum_{n=0}^{\infty} e^{-\frac{4 \pi n \omega(M-n \hbar \omega)}{\hbar}}\left|n_{\text {out }}^{L}\right\rangle \otimes\left|n_{\text {out }}^{R}\right\rangle .
$$

However, this yielded a contraditctory result to PW's model. No matter what particular tunneling process, the effect of backreaction in PW's result can be summarized as follows.

$$
\begin{aligned}
& -\frac{2 \pi\left(E-\Phi_{H}(Q) q-\Omega_{H}(M, J) j\right)}{\kappa(M, Q, J)}=-2 \pi \int_{\{0,0,0\}}^{\{E, q, j\}} \frac{d E^{\prime}-\Phi_{H}(Q) d q^{\prime}-\Omega_{H}(M, J) d j^{\prime}}{\kappa(M, Q, J)} \\
& \rightarrow-2 \pi \int_{\{0,0,0\}}^{\{E, q, j\}} \frac{d E^{\prime}-\Phi_{H}\left(Q-q^{\prime}\right) d q^{\prime}-\Omega_{H}\left(M-E^{\prime}, J-j^{\prime}\right) d j^{\prime}}{\kappa\left(M-E^{\prime}, Q-q^{\prime}, J-j^{\prime}\right)} \\
& =2 \pi \int_{\{M, Q, J\}}^{\{M-E, Q-q, J-j\}} \frac{d M^{\prime}-\Phi_{H}\left(Q^{\prime}\right) d Q^{\prime}-\Omega_{H}\left(M^{\prime}, J^{\prime}\right) d J^{\prime}}{\kappa\left(M^{\prime}, Q^{\prime}, J^{\prime}\right)}
\end{aligned}
$$

in which $E, q, j$ is respectively the energy, charge, and angular momentum of outgoing particles. It is simplest for a Schwarzchild black hole.

$$
\begin{aligned}
-\frac{2 \pi E}{\kappa(M)} \longrightarrow & -2 \pi \int_{0}^{E} \frac{d E^{\prime}}{\kappa\left(M-E^{\prime}\right)}=2 \pi \int_{M}^{M-E} \frac{d M^{\prime}}{\kappa\left(M^{\prime}\right)} \\
& =2 \pi \int_{M}^{M-E} \frac{d M^{\prime}}{\frac{1}{4 M^{\prime}}}=-8 \pi M E+4 \pi E^{2}
\end{aligned}
$$

So, the compatible way to correct by hand is redefining $-8 \pi n M \hbar \omega \longrightarrow-8 \pi n M \hbar \omega+4 \pi n^{2} \hbar^{2} \omega^{2}$. The quantum state should be

$$
|\psi\rangle=N \sum_{n=0}^{\infty} e^{-\frac{4 \pi n M \omega-2 \pi n^{2} \hbar \omega^{2}}{\hbar}}\left|n_{\text {out }}^{L}\right\rangle \otimes\left|n_{\text {out }}^{R}\right\rangle .
$$

In the simple harmonic oscillator canonical ensemble model, three assumptions are needed at the present stage: (i) The coincidence between canonical ensemble and PW's framework can go beyond the single particle state; (ii) The black hole is treated as a heat bath with minus heat capacity in flat 
spacetime; (iii) No mixture between different modes. Assumption $\mathrm{i}$ is the most fundamental one in our model, and (ii) and (iii) are not. Assumption ii can be changed. One can take the effect from curved spacetime into account, then go further to study the spectra in Schwarzchild-AdS spacetime. If this is the case, the mode summation cannot be done by integrating in phase space like Expression (19). Reference [33] gives a systemic method to calculate the sum in spherical spacetime. However, the infinity red shift due to Killing horizon can only be cured by introducing a UV cut-off at present stage.

Assumption (iii) can also be relaxed, since it is just a working assumption. It is worth to study what the effect of the mixture term in the partition function is. Meanwhile, there is a more serious problem about the divergence of the sum. Fortunately, its Taylor series is well defined when $s=\lambda k_{B}^{2} T^{2} \ll 1$, such that we can calculate the flux order by order. However, if $s=\lambda k_{B}^{2} T^{2} \sim 1$, this expansion becomes invalid. The corresponding temperature is $T \sim \sqrt{\frac{\hbar c^{5}}{4 \pi G k_{B}^{2}}}$, which is close to Planck scale. This suggests that there is a huge change while the emission temperature is near Planck scale. Finding a regularization for the divergent sum may be important in further investigation.

Acknowledgments: This research is supported by the National Natural Science Foundation of China under Grant Nos. 11273009 and 11303006.

Author Contributions: Jinbo Yang and Jingyi Zhang conceived the idea. Jinbo Yang derived the formula. Tangmei He, Jinbo Yang and Jingyi Zhang analyzed the result. Jinbo Yang drafted the manuscript. Jingyi Zhang and Tangmei He revised the manuscript. All the authors have read and approved the final manuscript.

Conflicts of Interest: The authors declare no conflict of interest.

\section{References}

1. Hawking, S.W. Black hole explosions? Nature 1974, 248, 30-31.

2. Hawking, S.W. Particle Creation by Black Holes. Commun. Math. Phys. 1975, 43, 199-220.

3. Hartle, J.B.; Hawking, S.W. Path-integral derivation of black-hole radiance. Phys. Rev. D 1976, $13,2188$.

4. Hawking, S.W. Breakdown of predictability in gravitational collapse. Phys. Rev. D 1976, 14, 2460.

5. Hawking, S.W. Information loss in black holes. Phys. Rev. D 2005, 72, 084013.

6. Parikh, M.K.; Wilczek, F. Hawking Radiation as Tunneling. 1999, arxiv:hep-th/9907001.

7. Parikh, M.K. A Secret Tunnel Through The Horizon. 2004, arXiv:hep-th/0405160.

8. Parikh, M.K. Energy Conservation and Hawking Radiation. 2014, arXiv:hep-th/0402166.

9. Hemming, S. Keski-Vakkuri, E. Hawking radiation from AdS black holes. Phys. Rev. D 2001, 64, 044006.

10. Medved, A.J.M. Radiation via tunneling from a de Sitter cosmological horizon. Phys. Rev. D 2002, 66, 124009.

11. Alves, M.; Dolgov, A. A Simple Line Element for the Dilaton Gravity. Int. J. Mod. Phys. D 2001, 10, 575-578.

12. Zhang, J.; Zhao, Z. Hawking radiation via tunneling from Kerr black holes. Mod. Phys. Lett. A 2005, 20, 1673-1681.

13. Zhang, J.; Zhao, Z. New coordinates for Kerr-Newman black hole radiation. Phys. Lett. B 2005, 618, 14-22.

14. Liu, W.B. New coordinates for BTZ black hole and Hawking radiation via tunnelling. Phys. Lett. B 2006, 634, 541-544.

15. Wu, S.Q.; Jiang, Q.Q. Remarks on Hawking radiation as tunneling from the BTZ black holes. J. High Energy Phys. 2006, 3, doi:10.1088/1126-6708/2006/03/079.

16. Zhang, J.; Zhao, Z. Massive particles' black hole tunneling and de Sitter tunneling. Nucl. Phys. B 2005, 725, 173-180.

17. Zhang, J.; Zhao, Z. Hawking radiation of charged particles via tunneling from Reissner-Nordstrom black hole. J. High Energy Phys. 2005, 10, 055.

18. Zhang, J.; Zhao, Z. Charged particles' tunnelling from the Kerr-Newman black hole. Phys. Lett. B 2006, 638, $110-113$.

19. Zhang, J.; Zhao, Z. Massive particles' Hawking radiation via tunneling. Acta Phys. Sin. 2006, 55, 3796-3798. (In Chinese)

20. Zhang, J.; Zhao, Z. Information loss in black hole evaporation. Mod. Phys. Lett. A 2006, 21, 1865-1868.

21. Zhang, J.; Fan, J.-H. Hawking radiation via tunnelling from general stationary axisymmetric black holes. Chin. Phys. 2007, 16, 3879. 
22. Zhang, J.; Fan, J.-H. Tunnelling effect of charged and magnetized particles from the Kerr-Newman-Kasuya black hole. Phys. Lett. B 2007, 648, 133-138.

23. Zhang, J. Tunneling effect of charged and magnetized particles from the Reissner-Nordstroem black hole with magnetic charges. Mod. Phys. Lett. A 2007, 22, 1821-1828.

24. Zhang, J. Black hole quantum tunnelling and black hole entropy correction. Phys. Lett. B 2008, 668, 353-356.

25. Zhang, J. Black hole entropy, log correction and inverse area correction. Phys. Lett. B 2009, 675, 14-17.

26. Zhang, J. Entropy correction of BTZ black holes in a tunneling framework. Sci. China Phys. Mech. Astron. 2010, 53, 1427.

27. Jiang, Q.-Q.; Wu, S.-Q.; Cai, X. Hawking radiation as tunneling from the Kerr and Kerr-Newman black holes. Phys. Rev. D 2006, 73, 064003.

28. Banerjee, R.; Majhi, B.R. Quantum tunneling and back reaction. Phys. Lett. B 2008, 662, 62-65.

29. Banerjee, R.; Majhi, B.R.; Samanta, S. Noncommutative Black Hole Thermodynamics. 2008, arXiv:0801.3583.

30. Banerjee, R.; Majhi, B.R. Quantum Tunneling Beyond Semiclassical Approximation. 2008, arXiv:0805.2220.

31. Kar, S. Tunneling between de Sitter and anti de Sitter black holes in a noncommutative D3-brane formalism. 2006, arxiv:hep-th/0607029.

32. Modak, S.K. Backreaction due to quantum tunneling and modification to the black hole evaporation process. Phys. Rev. D 2014, 90, 044015.

33. Hooft, G. On the quantum structure of a black hole. Nucl. Phys. B 1985, 256, 727-745.

(C) 2016 by the authors; licensee MDPI, Basel, Switzerland. This article is an open access article distributed under the terms and conditions of the Creative Commons Attribution (CC-BY) license (http://creativecommons.org/licenses/by/4.0/). 